\title{
COMPARISON OF HIGH ENERGY NEUTRON-NUCLEUS TOTAL CROSS SECTIONS WITH THEORY
}

\author{
C.A. AYRE and M.J. LONGO \\ University of Michtgan, Ann Arbor, Michtgan, USA
}

Received 15 March 1976

\begin{abstract}
A recent paper by Julius purports to show that previous estimates of the effect of inelastic screening on neutron-nucleus total cross sections are much too large. However, we show that most of this discrepancy is due to his using cross sections for diffractive $\mathrm{N}^{*}$ production which are much too small. The rest may be due to the assumptions in his model. We also emphasize that experımental support for the inelastic screening corrections does not rely on a specific choice of nuclear radiı
\end{abstract}

In a recent paper [1] Julius concludes that previous calculations $[2,3,4]$ seriously overestimate the effect of inelastic screening on the total cross sections of high-energy neutrons on nuclei. Inelastic screening results from diagrams such as that in fig. 1(c) in which the intermediate $N^{*}$ is an excited state of the nucleon. Diagrams such as those in figs. 1(a) and 1(b) are already taken into account in the conventional Glauber theory [5].

Julius used the coupled-channel eikonal approach of Bochmann, Kofoed-Hansen, and Margolis [6] but carries it out to all orders. The difficulty is that such a calculation requires knowledge of the amplitudes $f_{j k}(0)$ for processes like $\mathrm{N}_{j}^{*}+\mathrm{N} \rightarrow \mathrm{N}_{k}^{*}+\mathrm{N}$ where $\mathrm{N}_{j}^{*}$ and $N_{k}^{*}$ are excited states of the nucleon. Experimentally there are some limited data for $\mathrm{N}+\mathrm{N} \rightarrow \mathrm{N}_{j}^{*}+\mathrm{N}$, but little else is known about the amplitudes or their relative phases.

In his calculation Julius assumed that the cross section for $\mathrm{N}+\mathrm{N} \rightarrow \mathrm{N}^{*}+\mathrm{N}$ at $t=0$ is given by

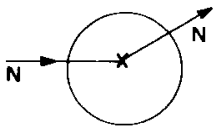

(a)

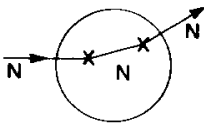

(b)

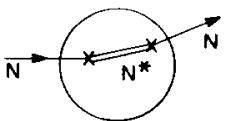

(c)
Fig. 1. (a) First-order diagram for elastic scattering of a nucleon on a nucleus. (b) Second-order diagram in which the intermediate state is a nucleon ("elastic screenıng"). (c) Secondorder diagram in which the intermediate state is a higher mass state than the nucleon ("inelastic screening").

$$
\frac{\mathrm{d}^{2} \sigma(t=0)}{\mathrm{d} t \mathrm{~d}_{\mathrm{x}}^{2}}=\frac{2.9}{M_{\mathrm{x}}^{2}} \quad \mathrm{mb} / \mathrm{GeV}^{4}
$$

where $M_{\mathrm{x}}$ is the mass of the $\mathrm{N}^{*}$. This expression is compared with experimental data [7] $\neq^{1}$ in fig. 2 . It disagrees markedly with the data for $M_{\mathrm{x}}^{2}<3$ $\mathrm{GeV}^{2}$, and even for larger masses is well below the

${ }^{1}$ The data of Akimov et al. [7] were extrapolate to $t=0$ using the quoted exponential slopes. They were also corrected for screening in the deuteron by multiplying by $\left(\sigma_{\mathrm{T}}^{\mathrm{pp}} / \sigma_{\mathrm{T}}^{\mathrm{pd}}\right)^{2}$, as Akımov et al. suggest.

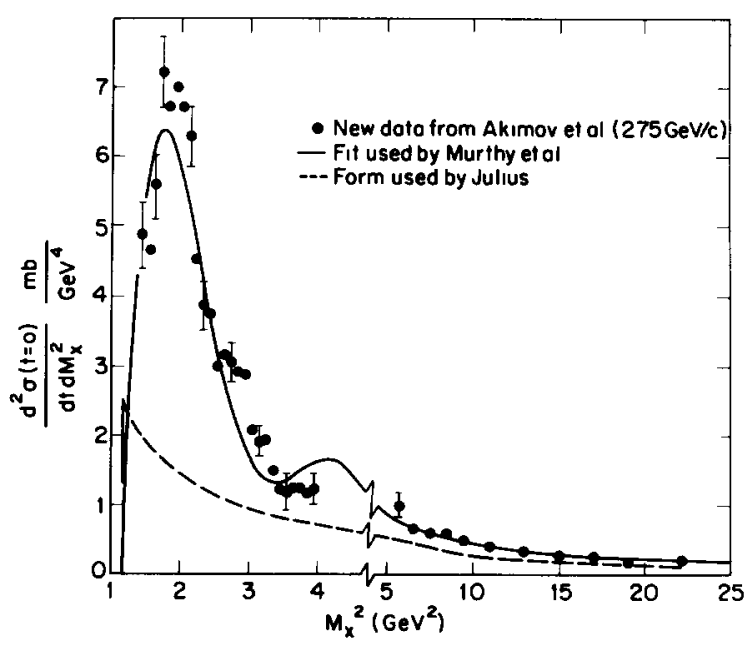

Fig. 2. Cross sections for $\mathrm{N}+\mathrm{N} \rightarrow \mathrm{N}+\mathrm{X}$ extrapolated to $t=0$. The solid curve is a fit to earlier Fermilab data. The dashed curve is the form used by Julius 
data. Karmanov and Kondratyuk [3], in the1r calculation of inelastıc screening before Fermilab data were available, used the form in eq. (1) for $M_{\mathrm{x}}^{2}>4$ $\mathrm{GeV}^{2}$, but below $4 \mathrm{GeV}^{2}$ they used a much larger cross section based on data from Brookhaven and CERN. In our calculation (Murthy et al. [4]) we used the same formalısm as Karmanov and Kondratyuk with early data on $\mathrm{N}^{*}$ production from Fermilab.

Julius notes that his calculation carried out to second order - as was done by Karmanov and Kondratyuk $(\mathrm{K}+\mathrm{K})$ - gives considerably smaller corrections to the cross sections calculated from Glauber theory than those of $\mathrm{K}+\mathrm{K}$ and Murthy et al. The reason for this discrepancy is clear from fig. 2. The correction to the calculated cross sections due to inelastic screening is roughly proportional to the area under these curves. The solid curve in fig. 2 is the form used by Murthy et al. It was fitted to early Fermilab data and is in good agreement with the more recent data [1], while the expression used by Julius grossly underestımates the cross section at all $M_{\mathrm{x}}^{2}$.

In fig. 3 we compare inelastic screening corrections for lead calculated with $\mathrm{d}^{2} \sigma / \mathrm{d} t \mathrm{~d} M_{\mathrm{x}}^{2}=2.9 / M_{\mathrm{x}}^{2}$, the form used by Julius, and those calculated with the fit used by Murthy et al. [4]. The curves labelled $K+K$ in fig. 3 are calculated from the theory of Karmanov and Kondratyuk [3]. In this calculation we used a WoodsSaxon nuclear shape with nuclear parameters, nucleon-

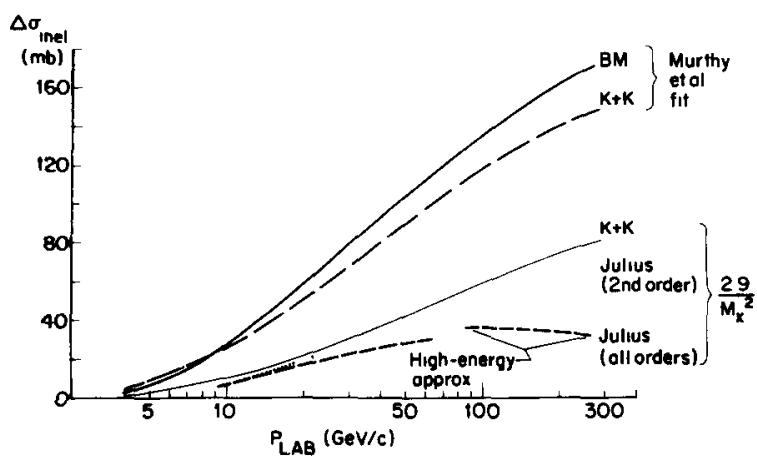

Fig. 3. Inelastic screening correction for lead calculated using the solid curve or dashed curve from fig. 2 The $\mathrm{K}+\mathrm{K}$ curves were calculated from the theory of Karmanov and Kondratyuk. The Bochmann-Margolıs (BM) calculation described in the text gives a similar result. The lowest curve is Julius' calculation to all orders; above $70 \mathrm{GeV} / c$ it was calculated using a high-energy approximation. nucleon total cross section, and other parameters as given in Murthy et al. As expected, the curves for $\Delta \sigma$ calculated from the solid curve in fig. 2 give a much larger correction. The lower $\mathrm{K}+\mathrm{K}$ curve in fig. 3 agrees well with Julius' calculation carried out to second order as it should.

From fig 3 we conclude that Julius' results for $\Delta \sigma_{1 \text { nel }}$ should be multiplied by a factor of approximately 2.1 to correct for his unrealistic choice for the $\mathrm{N}^{*}$ production cross sections. This removes much of the discrepancy between his results and earlier work. There is still some discrepancy with his calculation carried out to all orders (the lowest curve in fig. 3) at energies $\gtrsim 50 \mathrm{GeV} / c$. In order to carry out this calculation Julius had to make several important assumptions concerning the amplitudes $f_{i k}$ (defined above) and their relative phases. His assumptions, though perhaps reasonable in view of the lack of experimental information, are ad hoc. His choice for the amplitudes has the effect of giving especially large contributions to $\Delta \sigma_{\text {inel }}$ from higher order diagrams. Other equally reasonable assumptions for the amplitudes can give screening corrections as large or larger than the $\mathrm{K}+\mathrm{K}$ calculation in which only diagrams with one intermediate $\mathrm{N}^{*}$ were considered. In partıcular, Henyey [8], on the basis of the triple Regge model, finds for the amplitudes $\left|f_{j k} / k\right|^{2}=\left(0.24 / m_{j} m_{k}\right)^{2}$ $\mathrm{mb} / \mathrm{GeV}^{2}$ where $m_{l}$ and $m_{k}$ are the masses of the intermediate $\mathrm{N}^{*}$ 's in units of the nucleon mass $\neq^{2}$. This is to be compared with Julius' assumption [1] that $\left|f_{j k} / k\right|^{2}=0.92 /\left(\left|m_{j}^{2}-m_{k}^{2}\right|+1\right)$. Henyey's model has the effect of giving a much weaker coupling between the high mass states.

In his calculation Julius used the coupled-channel approach of Bochmann et al. [6]. We have used the same formalism with different assumptions to calculate $\Delta \sigma_{\text {inel }}$. In our calculation the missing mass spectrum given by the solid curve in fig. 2 was divided up into 20 to 30 bins extending from threshold to the kınematic limit $M_{\mathrm{x}}^{2} \lesssim s$. Each of the bins was considered as an " $\mathrm{N}$ "" whose production cross section was the area of the bin. In addition,

(i) We assume that the amplitudes for $\mathrm{N}_{j}^{*}+\mathrm{N} \rightarrow$ $\mathrm{N}_{j}^{*}+\mathrm{N}$ are the same as for $\mathrm{N}+\mathrm{N} \rightarrow \mathrm{N}+\mathrm{N}$. (Julius made the same assumption.)

$\ddagger^{2}$ It is assumed that $m_{j}^{2}>m_{\mathrm{N}}^{2}$. The mass interval is taken as $\Delta m=m_{\mathrm{N}}$ as was done by Julius. 
(ii) The phases for $\mathrm{N}+\mathrm{N} \rightarrow \mathrm{N}+\mathrm{N}_{\mathrm{f}}^{*}$ are assumed to be the same as that for $\mathrm{N}+\mathrm{N} \rightarrow \mathrm{N}+\mathrm{N}$.

(iii) In Henyey's model described above the $f_{j k}$ are small if both masses are large. In the spirit of this model we assume that the intermediate $\mathrm{N}^{*}$ 's couple only to the nucleon, not to each other.

The result of our calculation for lead is shown in fig. 3 as the curve labelled BM. This curve is very close to the $\mathrm{K}+\mathrm{K}$ curve calculated with the same $\mathrm{N}^{*}$ production cross sections and does not fall off at high momenta as does Julius' calculation to all orders. Thus it is clear that a calculation of $\Delta \sigma_{\text {inel }}$ to higher orders involves considerable uncertainty because of the assumptions that must be made about the amplitudes. The experimental data, as summarized in Murthy et al. [4], are in excellent agreement with the second order calculation of Karmanov and Kondratyuk [3]. This suggests that the contributions from the higher orders tend to cancel. Furthermore the second order calculation should be adequate for lighter nuclei such as carbon to at least $100 \mathrm{GeV}$ (see ref. [1]). The data for carbon below $100 \mathrm{GeV}$ give strong evidence for the need for the inelastic corrections [4].

Juluus concludes that the experimental data [4] could have been fitted without inelastic screening corrections if different radii were used, in particular, the radii used by Franco [9] in a comparison of Glauber theory with lower energy data. Julius' conclusion is incorrect for several reasons:

1) As we stressed in Murthy et al. [4], evidence of the need for inelastic screening corrections comes from the energy dependence of the cross sections, not their overall magnitude. Our conclusion was therefore independent of the choice of nuclear radii.

2) Our choice of radii generally give calculated cross sections which agree with the low energy data as well as or better than those used by Franco.

3) Julius has grossly overestimated the change in the calculated cross sections that would result from the use of Franco's radii (and skin thicknesses).

To illustrate the effect of using Franco's radii, we compare in fig. 4 the data for copper and lead with theoretical calculations made using a Woods-Saxon nuclear shape with Franco's radii and skin thicknesses. The solid curves are the theory with inelastic screening calculated using the $\mathrm{K}+\mathrm{K}$ theory with the solid curve from fig. 2; the dashed curves are from Glauber theory without inelastic screening [5,9]. For copper

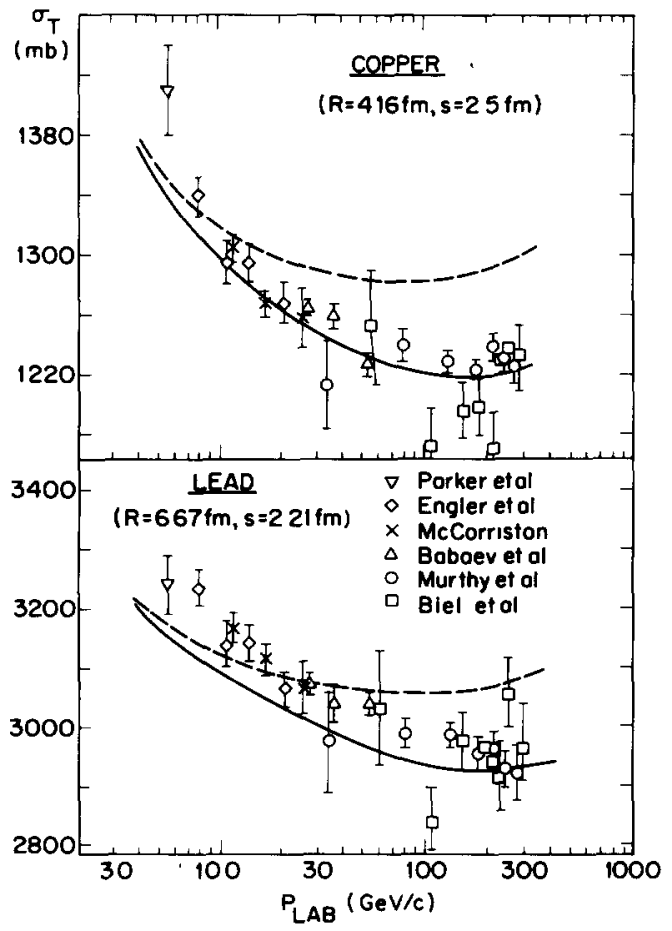

Fig. 4. Comparison of calculated neutron total cross sections with data above $4 \mathrm{GeV} / c$. The solid curves are the theory with inelastic screening, the dashed curves do not include inelastic screenıng. The radıl and skin thicknesses are those used by Franco [9] in his fit to the low energy data. Other parameters are the same as those given in Murthy et al. [4].

the theory with inelastic screening agrees well with the data at all momenta while the dashed curve misses the high energy data by many standard deviations. For lead the solid curve lies about $1.5 \%$ lower than the data, but it has the correct energy dependence; the dashed curve lies below the data at low momenta and above it at high momenta. However, Franco's calculated cross sections for lead tend to lie below the data for neutron momenta $<10 \mathrm{GeV} / c$ where inelastic screening is negligible. In any case an increase of $0.05 \mathrm{fm}$ in the rms radius for lead would make the solid curve agree very well with the data. This is well within the uncertainty in nuclear radii $\neq 3$.

\footnotetext{
$\neq^{3}$ Franco [9] used nuclear radii determıned from electromagnetic interactions. It would be surprising of the effective radil for strong interactions were not slightly larger.
} 
Also included in fig. 4 are recent data of Biel et al. [10] from Fermilab. These tend to be slightly lower than the data of Murthy et al. [4]. However, if taken at face value the data of Biel et al. would suggest that even larger inelastic screening corrections are needed.

Helpful conversations with Professors G. Kane and B. Margolis are gratefully acknowledged. We are especially indebted to Dr. T. McCorriston for many of the computer programs used for the calculations and to Professor F. Henyey for his theoretical assistance.

\section{References}

[1] D. Julius, Phys. Lett. 60B (1975) 58.

[2] V. Gribov, Soviet Phys. JETP 30 (1970) 709.

[3] V. Karmanov and L. Kondratyuk, JETP Lett (Soviet Phys.) 18 (1973) 266.

[4] P.V.R. Murthy et al., Nucl. Phys. B92 (1975) 269.

[5] R.J. Glauber, Phys. Rev. 100 (1955) 242;

V. Franco and R.J. Glauber, Phys. Rev. 142 (1966) 1195.

[6] G. Bochmann, O. Kofoed-Hansen and B. Margolis, Phys. Lett. 33B (1970) 222.

[7] Y. Akımov et al., Phys Rev. Lett 35 (1975) 763.

[8] F. Henyey, private communication.

[9] V. Franco, Phys. Rev. C6 (1972) 748.

[10] J. Biel et al., University of Rochester Rep't. UR-560 C00 3065-135, submitted to Phys. Rev. Lett. Comments. 